\title{
Editorial for the special issue: Digital Economy and E-commerce Technology
}

\author{
Christos K. Georgiadis • Emmanouil Stiakakis • \\ A. Ravi Ravindran
}

Published online: 28 October 2011

(C) Springer-Verlag 2011

The aim of this special issue is to provide insights into the use of Operational Research (OR) applications in the context of the digital economy and electronic commerce (e-commerce) technologies.

The term "digital economy" (or Internet economy) refers to all the economic and social activities that are enabled by the Internet and the other Information and Communication Technologies (ICTs). The digital economy is not a totally new economy, but rather the whole of new ways to accomplish the usual economic and social activities through the use of ICTs. In the past, there were numerous applications of OR in the micro- and macro-economy; nowadays OR also seems to offer a great potential to the further development of the digital economy. There are many issues in the context of the digital economy which need to be studied to a greater extent. Some of them are indicatively mentioned in the following lines: (1) the modeling of the pricing on the Internet, (2) the assessment of the economic changes arising from the use of open-source software, (3) the assessment of the economic implications of software piracy, (4) the measurement of digital inequalities in the usage of ICTs, and (5) the measurement of productivity and efficiency in the Internet economy. A reasonable question that rises is whether the

C. K. Georgiadis $(\bowtie) \cdot$ E. Stiakakis

Department of Applied Informatics, University of Macedonia,

Economic and Social Sciences, 156 Egnatia Str., 54006 Thessaloniki, Greece

e-mail: gxri@acm.org

URL: http://macedonia.uom.gr/ geor

E. Stiakakis

e-mail: stiakakis@uom.gr

URL: http://users.uom.gr/ stiakakis/

A. R. Ravindran

Pennsylvania State University, 352 Leonhard Building,

University Park, PA 16802, USA

e-mail: aravi@psu.edu

URL: http://www.ie.psu.edu/FacultyStaff/Faculty/FacultyProfile.cfm?FacID=21 
existing OR methodologies and tools can deal with these issues. If we analyzed, for instance, the issue (5), we could find out that the productivity increase due to ICTs could not be measured using traditional methodologies. This has been recorded as one of the reasons that explained the so called "productivity paradox", i.e. the fact that the models used to measure productivity failed to find any positive linkage between ICT investment and total factor productivity, which obviously exists. Therefore, there is the need for new OR tools adjusted to the particularities of digital technologies and the specific characteristics of the digital economy. OR academics and researchers should prepare for a future in which the digital economy will be a major part of the whole economic and social activity.

Given the interdisciplinary nature of e-commerce, OR methodologies and tools have been extensively studied and used in several features of e-commerce in past years. The success behind many companies can be traced to OR embedded within their patented algorithms. Logistics is a major area where OR mechanisms can make the difference. Undoubtedly, a real challenge of many companies is the minimization of the time between the placement of an electronic order and the actual shipping of goods to the customer. Supply chain optimization refers to the optimization of inventory across the entire supply chain (factory-warehousedistributors-stores). The goal is the calculation of the proper volume of safety stocks at different levels. The forecasting of inventory of today's supply chain optimization mechanisms varies from a plain probability distribution to a complicated stochastic procedure.

Auctions and negotiations is another important area, as the Internet provides transparency and several efficiencies: e-auctions may remove geographical/time limitations and thus may attract larger numbers of bidders. Typically, when a seller declares that he has an item for sale, various buyers appear and bid for it by announcing a bid price. As all bidders know the bid price of other bidders, they hopefully offer a higher price to surpass the earlier bid. Generally, an auction is for a limited time and at the end of the time limit, the seller sells the item to the highest bidder. In reverse auction, it is the buyer (e.g., a procurement manager) who initiates the auction for all the sellers to bid. This type of auction facilitates the process of buying items from multiple suppliers. Multi-criteria decision making approaches are used to evaluate the differing constraints of bidders and buyer (or seller), as well as linear programming to solve the formulated equations' system. OR tools can also be used for auction data analysis in order to improve the behavior of future auctions.

Recommending possible buy items to prospective customers is a research area which indicates also the importance of OR to e-commerce. Business researchers promote the need for one-to-one marketing (treating each customer individually in order to capture customer loyalty). Recommendation systems provide forecasts and estimations that help the user in evaluating items. Recommendation mechanisms may reduce dramatically the amount of information the user must search through to find the items they are interested in. They are capable to incorporate the opinions or experiences of a community and produce for the user a mapping of scores to items, a ranking of items or a set of recommended items. To develop the best recommendation, rule-based practices are usually combined with filtering methods. Advanced filtering approaches utilize algorithms to analyze user profiles. Such a representative 
approach is the collaborative filtering: as an alternative of locating objects comparable to those a user was fond of in the past (the process of the content-based filtering approach), collaborative filtering builds up recommendations by discovering users with similar inclinations. The collection of users' opinion on a set of objects is used to structure like-minded peer groups. These groups are used to predict a particular user's concern in an item. Similarly, cluster methods that split the customers' base into many segments using a classification scheme are also exploited. A potential user is classified into one of the groups and recommendations are formulated based on the buying pattern of the users in that segment. Among the techniques of OR, that recommendation algorithms utilize, is network optimization (to capture resemblance and identify clusters).

Finally, in the broad area of customer relationship management, the service quality perspective is a popular topic that OR professionals have dealt in e-commerce area. Typical examples are studies which develop research models consisting of hypothesized relationships among potential factors, works which apply quantitative techniques-such as factor analysis, structural equation modeling (SEM), etc.- - in order to discover the aspects of perceived service quality (taking into account consumer behavior, technology acceptance, and service acceptance models) in both static and mobile environments, and research works which employ multi-criteria analysis to evaluate the quality dimensions associated with e-services.

The research works that are presented in this special issue have been chosen using the following criteria: being representative of the state-of-the-art issues of concern on certain aspects of digital economy and e-commerce, and at the same time being able to provide concrete examples of the usefulness of OR mechanisms.

The first paper by Mendoza and Ventura proposes a mixed-integer nonlinear programming model to allocate appropriately order quantities to the selected set of suppliers, while taking into account inventory, purchasing, and transportation expenses under quality constraints and suppliers' capacity. The proposed model can be extended to unravel dynamic inventory problems with time varying demand for a fixed number of periods using a time-expanded network.

In the second paper, Gujar and Narahari present and analyze an optimal combinatorial auction for a buyer seeking to procure multiple units of multiple items in the presence of capacitated and single minded sellers (each seller is bidding on exactly one bundle). The proposed mechanism minimizes the asking price of procurement while satisfying Bayesian incentive compatibility and interim individual rationality.

The next paper by Mandala, Kumara, Rao, and Albert focuses on the detection of community structures in social networks. Understanding the structure of social networks is an important issue for customizing and personalizing e-commerce applications. An approach based on ant colony optimization is proposed to reveal the extremely dense sub-graphs (the segments of the social network) in a given graph by maximizing the modularity measure (a representation of the quality of a network division).

In the fourth paper by Grimaila and Badiru, the application of a simulation-based hybrid analytic dynamic forecasting methodology is presented. More specifically, the methodology uses qualitative subjective assessments with the aid of experts at 
multiple levels of the organization in order to provide the decision maker with an ongoing recommendation of the best contingency measures.

The special issue closes with the paper of Loukis, Soto-Acosta, and Pazalos, who investigate the impact of the adoption of an e-business strategy on ICT and non-ICT assets, as well as on business processes and performance. Advanced quantitative techniques of SEM have been employed.

We would like to truthfully thank all the authors and reviewers for their productive contribution to this special issue of Operational Research: An International Journal. 